\title{
6
}

\section{ANALYTICAL AND SimUlation RESUltS}

\subsection{Small-Signal Response Without Supplementary Control}

As discussed in Section 5.6, the complete A-matrix equations containing all of the singlegenerator terms and the cross-relationship terms are not directly available. The format of the Amatrix is known, however, to give the terms for each generator in the following order: 1, 2, 12, and 11. The "Base System" is the test system without any supplementary control on the machines (PSSs), but includes the simple-gain excitation model, the speed governor and terminal voltage controllers. The size of the A-matrix is then $40 x 40$, or 10 state variables per machine. There are the six machine variables, plus one from the terminal voltage controller and three from the speed governor.

The "oscillation profile" of the power system is simply the information garnered from the eigenanalysis. The oscillatory modes are identified, and their frequencies and damping factors are calculated. Next, probable local and inter-area modes are identified by looking at the magnitude of the participation factors of each mode with respect to the rotor angle, yielding the rotor angle modes. Once the rotor-angle modes are identified, the mode shape determines if the particular modes are local or inter-area. For the base system, there are three rotor angle modes, summarized in Table 6.1.

\begin{tabular}{|c|c|c|c|c|}
\hline Mode & $\begin{array}{c}\text { Frequency } \\
\text { Hz }\end{array}$ & $\begin{array}{c}\text { Damping } \\
\text { Ratio }\end{array}$ & $\begin{array}{c}\text { Dominant } \\
\text { States }\end{array}$ & $\begin{array}{c}\text { Mode } \\
\text { Shape }\end{array}$ \\
\hline$-0.7335 \pm \mathrm{j} 6.8457$ & 1.0895 & 0.1065 & $\Delta \omega \& \Delta \Theta, 1 \& 2$ & 1 vs 2 \\
$-0.7610 \pm \mathrm{j} 7.0415$ & 1.1207 & 0.1074 & $\Delta \omega \& \Delta \Theta, 11 \& 12$ & 11 vs 12 \\
$0.0217 \pm \mathrm{j} 3.8025$ & 0.6052 & -0.0057 & $\Delta \omega \& \Delta \Theta$, all 4 & 1,2 vs 12,11 \\
\hline
\end{tabular}

Table 6.1: Test System Base Case Oscillation Profile 


\begin{tabular}{|c|c|c|c|c|c|c|c|c|c|c|}
\hline \multicolumn{11}{|c|}{ Mode 15} \\
\hline GEN & $\lambda_{f}$ & $\lambda_{d}$ & $\lambda_{\mathrm{Q} 1}$ & $\lambda_{\mathrm{Q} 2}$ & Vc & Tm & Tm & Tm & $\omega$ & $\theta$ \\
\hline 1 & 0.0005 & 0.0001 & 0.0007 & 0.0005 & 0.0 & 0.0 & 0.0 & 0.0 & 0.0062 & 0.0055 \\
\hline 2 & 0.0028 & 0.0002 & 0.0019 & 0.0012 & 0.0002 & 0.0002 & 0.0001 & 0.0 & 0.0167 & 0.0190 \\
\hline 12 & 0.0491 & 0.0109 & 0.0852 & 0.0554 & 0.0028 & 0.0 & 0.0 & 0.0 & 0.5921 & 0.5808 \\
\hline 11 & 0.0702 & 0.0012 & 0.0426 & 0.0277 & 0.0052 & 0.0 & 0.0 & 0.0 & 0.4674 & 0.4843 \\
\hline \multicolumn{11}{|c|}{ Mode 17} \\
\hline GEN & $\lambda_{f}$ & $\lambda_{d}$ & $\lambda_{\mathrm{Q}_{1}}$ & $\lambda_{\mathrm{Q} 2}$ & Vc & Tm & $\mathrm{Tm}$ & $\mathrm{Tm}$ & $\omega$ & $\theta$ \\
\hline 1 & 0.0206 & 0.0010 & 0.0034 & 0.0027 & 0.0008 & 0.0 & 0.0 & 0.0 & 0.2351 & 0.3064 \\
\hline 2 & 0.0448 & 0.0026 & 0.0079 & 0.0064 & 0.0019 & 0.0055 & 0.0023 & 0.0009 & 0.1307 & 0.1885 \\
\hline 12 & 0.0110 & 0.0013 & 0.0137 & 0.0110 & 0.0004 & 0.0 & 0.0 & 0.0 & 0.2930 & 0.2294 \\
\hline 11 & 0.0024 & 0.0006 & 0.0130 & 0.0105 & 0.0 & 0.0 & 0.0 & 0.0 & 0.3556 & 0.2839 \\
\hline
\end{tabular}

Table 6.2: Participation Factor Magnitudes

From the oscillation profile, it is clear that one mode is unstable with a positive real part and negative damping. This mode is indeed a mode oscillating between groups of generators, $1 \& 2$ versus $11 \& 12$, as indicated by the mode shape. Recalling the topology of the system from Figure 5.5, it is possible to see that coherent regions or areas have formed on each side of the interconnecting tie-lines, leading to the relevant descriptive title of "inter-area" oscillation.

Determination of the dominant states is performed by the examination of the participation factors (Section 3.3.2) of the states involved in the oscillation. For example, the participation factor magnitudes of two base case modes from Table 6.1 are given in Table 6.2, where the values for Modes 15 and 17 are shown. The $\lambda f, \lambda d$, etc. represent the state variables of the generator indicated (1,2, 12 or 11). For Mode 15, it can be seen that the participation factors for the speed $(\omega)$ and rotor angle $(\theta)$ of generators 11 and 12 have the largest magnitude. This indicates that these states have the greatest participation in this mode. This allows the classification of this mode as a rotor angle mode, or one of interest. At this point, the mode shape is not known and the modes may be local, inter-area or another type. For Mode 17, it can be seen that all four generators have the largest participation factors for the speed $(\omega)$ and rotor angle $(\theta)$. Indeed, this is another rotor angle mode, yet the type of mode is indeterminable at this point.

Once rotor angle modes have been identified using the participation factors, the mode shape (Section 3.3.3) can be quickly determined from the entries in the right eigenvector corresponding to the state variables involved in the mode, and helps to classify the mode as local or inter-area. The rotor angle right eigenvector entries for the two modes are shown in Table 6.3. It can be seen from the magnitude and sign of the real part of the eigenvector entries how the mode shape is determined. For Mode 15, GEN11 has the opposite sign as the other generator in its zone, indicating a local oscillation of this machine. This is the local mode GEN11 vs. GEN12 shown in Table 6.1. For Mode 17, however, it can bee seen that GEN1 and GEN2 swing coherently, as well as GEN12 and GEN 11. The sign of the real part of the eigenvector entries indicates that generators 1 and 2 swing against generators 12 and 11, yielding an inter-area oscillatory mode. This is, in fact, the inter-area mode shown in Table 6.1. 


\begin{tabular}{|c|c|}
\hline \multicolumn{2}{|c|}{ Mode 15 } \\
GEN & Eigenvector Entry \\
\hline 12 & $-0.0838 \pm \mathrm{j} 0.0900$ \\
11 & $0.5004 \pm \mathrm{j} 0.2822$ \\
\hline Mode 17 \\
GEN & Eigenvector Entry \\
\hline 1 & $-0.1327 \pm \mathrm{j} 0.5139$ \\
2 & $-0.1672 \pm \mathrm{j} 0.3555$ \\
12 & $0.1368 \pm \mathrm{j} 0.4349$ \\
11 & $0.1788 \pm \mathrm{j} 0.4802$ \\
\hline
\end{tabular}

Table 6.3: Right Eigenvector Entries For Two Oscillatory Modes

\subsection{Small-Signal Response With Local Feedback Supplementary Control}

A PSS is now added to the system to provide supplementary control. Its tuning is based on the method described in [26]. For the generator with the PSS, the excitation model changes to the ST1-Type controller; the other generators keep the simple-gain exciter. The speed governor and terminal voltage controllers remain the same for all of the generators. With the addition of the state variables and equations of the PSS and exciter for a single PSS, the size of the A-matrix is now 46x46, or 10 state variables per generator without PSSs, and 16 for a generator with a PSS.

The participation factors, combined with the eigenvalue and damping ratio, of the inter-area mode were used to investigate the controllability and observability to place the PSS control at its optimal location. All 15 possible combinations of placement of 1, 2, 3 and 4 PSSs were tested with two input signals, namely speed, $\Delta \omega=\omega_{\text {mach }}-\omega_{0}$, and accelerating power, $\Delta \mathrm{Pa}=\mathrm{P}_{\text {mech }}-\mathrm{P}_{\text {elec }}$. The optimal sites for the PSSs and PMUs were chosen based on a combination of damping on the inter-area mode and the distance of the eigenvalues from the imaginary axis. This combined approach helps eliminate the well-damped oscillatory modes that are distant from the imaginary axis, yet is still sensitive to the rather unstable poorly damped modes.

\begin{tabular}{|c|c|c|c|c|c|c|}
\hline $\begin{array}{c}\text { \# of } \\
\text { PSSs }\end{array}$ & $\begin{array}{c}\text { Location } \\
\text { GEN }\end{array}$ & Eigenvalue & $\begin{array}{c}\text { Frequency } \\
\text { Hz }\end{array}$ & $\begin{array}{c}\text { Damping } \\
\text { Ratio }\end{array}$ & $\begin{array}{c}\text { Dominant } \\
\text { Machines }\end{array}$ & $\begin{array}{c}\text { Mode } \\
\text { Shape }\end{array}$ \\
\hline 1 & 11 & $-3.1013 \pm \mathrm{j} 7.8069$ & 1.2425 & 0.3692 & $11 \& 12$ & $11 \mathrm{vs} 12$ \\
& & $-0.7370 \pm \mathrm{j} 6.8517$ & 1.0905 & 0.1069 & $1 \& 2$ & $1 \mathrm{vs} 2$ \\
& & $-0.4604 \pm \mathrm{j} 3.9781$ & 0.6331 & 0.1150 & all 4 & 1,2 vs 12,11 \\
\hline 2 & 12,11 & $-4.0990 \pm \mathrm{j} 8.6865$ & 1.3825 & 0.4268 & $11 \& 12$ & $11 \mathrm{vs} 12$ \\
& & $-0.7457 \pm \mathrm{j} 6.8423$ & 1.0890 & 0.1083 & $1 \& 2$ & 1 vs 2 \\
& & $-0.3978 \pm \mathrm{j} 2.5276$ & 0.4023 & 0.1555 & all 4 & 1,2 vs 12,11 \\
\hline 3 & $11,12,1$ & $-4.0893 \pm \mathrm{j} 8.6891$ & 1.3829 & 0.4258 & $11 \& 12$ & $11 \mathrm{vs} 12$ \\
& & $-2.8634 \pm \mathrm{j} 7.6318$ & 1.2146 & 0.3513 & $1 \& 2$ & 1 vs 2 \\
& & $-0.9374 \pm \mathrm{j} 3.0160$ & 0.4800 & 0.2968 & all 4 & 1,2 vs 12,11 \\
\hline
\end{tabular}

Table 6.4: Oscillation Profile of Speed Input 
The performance of both controllers based on input signals of speed deviation $(\Delta \omega)$ and accelerating power $(\Delta \mathrm{Pa})$ were similar. The tests involving the $\Delta \omega$ signal were used to confirm the results reported in [22]. The best results of 1,2, and 3 PSSs with the $\Delta \omega$ input signal for the three modes previously discussed are summarized in Table 6.4. The first column lists the number of PSSs in the system while the second column gives their location. Columns three and four contain the real and imaginary eigenvalue information, followed by the frequency and damping of that particular oscillatory mode. The next column indicates the dominant state variable influence garnered from the participation factors. Finally, the mode shape, as determined by the right eigenvector, is indicated. Recalling Table 6.1, there are two local oscillatory modes of interest, GEN1 vs. GEN2 and GEN11 vs. GEN12, as well as one inter-area mode, GEN1\&2 vs. GEN12\&11. Recalling the topology of the system, two regions can be defined for the inter-area oscillation: Area One containing generators 1 and 2, and Area Two containing generators 11 and 12. These areas, together with "Ptie" defined as the power flowing on the lines from Area One to Area Two into Bus 13, will be used in the following discussions.

A complete oscillation profile for the accelerating power input PSS is given in Table 6.5, with the same layout as Table 6.2. While the accelerating power as a local feedback variable was equally investigated in early PSS studies, its use in a remote feedback manner has not been previously addressed in any reported literature. This work is then the first look at this control signal under these conditions. It should be noted that this thesis is focusing on the damping of any inter-area modes; hence, as the local modes remain stable throughout the simulations performed on this system, their analysis will not be performed.

\begin{tabular}{|c|c|c|c|c|}
\hline $\begin{array}{c}\text { \# of } \\
\text { PSSs }\end{array}$ & $\begin{array}{c}\text { Location } \\
\text { GEN }\end{array}$ & Eigenvalue & $\begin{array}{c}\text { Frequency } \\
\text { Hz }\end{array}$ & $\begin{array}{c}\text { Damping } \\
\text { Ratio }\end{array}$ \\
\hline 1 & 1 & $-0.2386 \pm \mathrm{j} 3.9931$ & 0.6355 & 0.0596 \\
& 2 & $-0.2113 \pm \mathrm{j} 3.8142$ & 0.6070 & 0.0553 \\
& $\mathbf{1 1}$ & $-\mathbf{0 . 4 8 6 2} \pm \mathrm{j} \mathbf{4 . 0 2 9 1}$ & $\mathbf{0 . 6 4 1 3}$ & $\mathbf{0 . 1 1 9 8}$ \\
& 12 & $-0.4453 \pm \mathrm{j} 4.2343$ & 0.6739 & 0.1046 \\
\hline 2 & $\mathbf{1 , 1 1}$ & $-\mathbf{0 . 6 0 7 3} \pm \mathrm{j} \mathbf{4 . 2 7 3 3}$ & $\mathbf{0 . 6 8 0 1}$ & $\mathbf{0 . 1 4 0 7}$ \\
& 1,12 & $-0.4903 \pm \mathrm{j} 4.4123$ & 0.7022 & 0.1104 \\
& 2,11 & $-0.5548 \pm \mathrm{j} 4.0897$ & 0.6509 & 0.1344 \\
& 2,12 & $-0.4816 \pm \mathrm{j} 4.2529$ & 0.6769 & 0.1125 \\
& 1,2 & $-0.2880 \pm \mathrm{j} 3.1283$ & 0.4979 & 0.0917 \\
& 11,12 & $0.3118 \pm \mathrm{j} 2.5061$ & 0.3989 & 0.1235 \\
\hline 3 & $1,2,11$ & $-0.9569 \pm \mathrm{j} 3.7390$ & 0.5951 & 0.2479 \\
& $1,2,12$ & $-0.7204 \pm \mathrm{j} 4.1479$ & 0.6602 & 0.1711 \\
& $\mathbf{1 1 , 1 2 , 1}$ & $-\mathbf{0 . 8 8 5 5} \pm \mathrm{j} \mathbf{3 . 1 1 1 9}$ & $\mathbf{0 . 4 9 5 3}$ & $\mathbf{0 . 2 7 3 7}$ \\
& $11,12,2$ & $-0.8109 \pm \mathrm{j} 3.2722$ & 0.5208 & 0.2405 \\
\hline
\end{tabular}

Table 6.5: Oscillation Profile for Accelerating Power Input

From Table 6.5, it can be seen that the optimal location of 1, 2 or 3 PSSs, as determined by the maximum damping ratio and distance from the imaginary axis of the eigenvalue, is GEN11, GEN11 and 1, and GEN11, 12, and 1 respectively. While the 3 PSS case does not have the 
maximum distance from the imaginary axis, the damping ratio is much greater and provides a more influential point for choosing this PSS site selection over the others.

Development of the observability and controllability indices was not possible due to the same explicit equation problem surrounding the A-matrix. As a result, information about the controllability and observability of the modes of interest was investigated through the use of the participation factors, which are a function of these indices. This is a similar analysis to one performed with residues (Section 3.4) and represents an approximation of a residue as defined in several articles dealing with PSS tuning [21-23]. For example, the original weighting of the rotor angle participation factors for the inter-area mode were 0.4949 and 0.5133 for areas One and Two, respectively. With the addition of a PSS at its optimal site on GEN11, as determined by damping ratio and eigenvalue location, the factors become 0.4577 and 0.6706 . It is possible to conclude that either the controllability or observability (or both) of the mode have increased in Area Two, where the PSS is located.

\subsection{Small-Signal Response With Remote Feedback Supplementary Control}

In the following, it is assumed that PMUs are placed at the output terminal of the generators involved in the remote feedback controller, and that the electrical powers, as well as the mechanical powers, are available in a synchronized manner. It is also assumed that the remote signal feedback structure has as input an ideal measurement transmission network without delay. Given these assumptions, the PSS input signal is formed by the local signals and remote signals combined in the following manner:

$$
\Delta \mathrm{Pa}=\mathrm{Pa}_{\text {local }}-\mathrm{Pa}_{\text {remote }}=\mathrm{Pm}_{\text {loc }}-\mathrm{Pe}_{\text {loc }}-\mathrm{Pm}_{\text {rem }}+\mathrm{Pe}_{\text {rem }}
$$

The opposite input signal, namely $\Delta \mathrm{Pa}=\mathrm{Pa}_{\text {remote }}-\mathrm{Pa}_{\text {local }}$, was also investigated. However, (6.1) provides the best control result. Since the interest is in damping the inter-area oscillation, the PSS at its optimal site, GEN11, is provided with the $\triangle \mathrm{Pa}$ signal from the better of the two sites in the other area, GEN1, as determined from the PSS placement tests displayed in Table 6.5. This is called the "1-PSS/2-PMU" configuration. This also follows the results of the application of two local-feedback PSSs also shown in Table 6.5, whose optimal sites would be the same two generators.

\subsubsection{Preliminary Tuning}

As a first approximation, the parameters for the PSS with remote variable feedback were determined based on those of the local feedback PSS. Analysis of the participation factors was again used to evaluate the tuning of the PSS with remote input signal feedback. This analysis is in substitution of that which might be performed with residues. In particular, the participation factors for area one and two rotor angle state variables become 0.4612 and 0.7992 , respectively, with the addition of the 1-PSS/2-PMU structure on GEN11. This is in contrast to values of 0.4577 and 0.6706 for the local feedback PSS alone. Once again, it can be concluded that there has been an increase of the controllability or observability of this mode in Area Two due to the 
controller action. In addition, the magnitude of the local feedback controller state participation increases from 0.1310 to 0.1599 with the addition of the remote feedback structure, representing an increase in the participation of the PSS on this mode with the additional 2-PMU structure.

With remote feedback input signal, the gain $\mathrm{K}_{\mathrm{S}}$ of the PSS was adjusted to obtain the best response of the controller. The gain can be determined once the other parameters have been fixed by varying its value over a wide range and selecting the value representing the best compromise [1]. Fittingly, the tuning for the local feedback signal provided a modest amount of performance in terms of the two goals of damping the inter-area oscillation and increasing the stability limits. Modifying the PSS gain $\mathrm{K}_{\mathrm{S}}$ realized a preliminary benefit of increasing the damping ratio to a maximum value of 0.1507 . Further increasing the gain does yield further placement away from the imaginary axis, but with a lesser damping ratio. A high gain usually precipitates instability, and at $\mathrm{K}_{\mathrm{S}}=30.0$ an unstable mode does appear; however, this is not the mode of interest. Table 6.6 enumerates the gain modification tests. Column one lists the different PSS gain values investigated with their corresponding eigenvalues, frequency and damping ratios. It can be seen that a value of $\mathrm{K}_{\mathrm{S}}=15.5$ provides the best results in terms of the goal of maximizing both the damping ratio and the distance from the stability limit of the eigenvalue. A plot comparing the location of the mode of interest under the base case of Ptie $=558 \mathrm{MW}$ without a PSS (AVRs only), with a PSS on GEN1 and with a 1-PSS/2-PMU system on GEN11 and GEN1, is shown in Figure 6.1. It is evident that the addition of remote feedback control (1-PSS/2-PMUs) pushes the unstable eigenvalue further away from the imaginary axis than the case of a single PSS with local feedback. While the mode location is not in itself a final indicator of the stability of a mode, for a single operating point a plot such as this does provide a comparative look at the different control structures under similar constraints.

\begin{tabular}{|c|c|c|c|}
\hline $\begin{array}{c}\text { Gain } \\
\mathbf{K}_{\mathbf{S}}\end{array}$ & Eigenvalue & $\begin{array}{c}\text { Frequency } \\
\mathbf{H z}\end{array}$ & $\begin{array}{c}\text { Damping } \\
\text { Ratio }\end{array}$ \\
\hline 11.3 & $-0.6483 \pm \mathrm{j} 4.3253$ & 0.6884 & 0.1482 \\
13.5 & $-0.6677 \pm \mathrm{j} 4.3926$ & 0.6991 & 0.1503 \\
14.0 & $-0.6706 \pm \mathrm{j} 4.4059$ & 0.7012 & 0.1505 \\
14.5 & $-0.6731 \pm \mathrm{j} 4.4185$ & 0.7032 & 0.1506 \\
15.0 & $-0.6752 \pm \mathrm{j} 4.4306$ & 0.7051 & 0.1507 \\
$\mathbf{1 5 . 5}$ & $-\mathbf{0 . 6 7 7 0} \pm \mathrm{j} 4.4420$ & $\mathbf{0 . 7 0 7 0}$ & $\mathbf{0 . 1 5 0 7}$ \\
16.0 & $-0.6785 \pm \mathrm{j} 4.4529$ & 0.7087 & 0.1506 \\
17.5 & $-0.6816 \pm \mathrm{j} 4.4825$ & 0.7134 & 0.1503 \\
20.0 & $-0.6837 \pm \mathrm{j} 4.5235$ & 0.7199 & 0.1494 \\
25.0 & $-0.6821 \pm \mathrm{j} 4.5832$ & 0.7294 & 0.1472 \\
\hline
\end{tabular}

Table 6.6: Remote Feedback Controller Gain Adjustment 


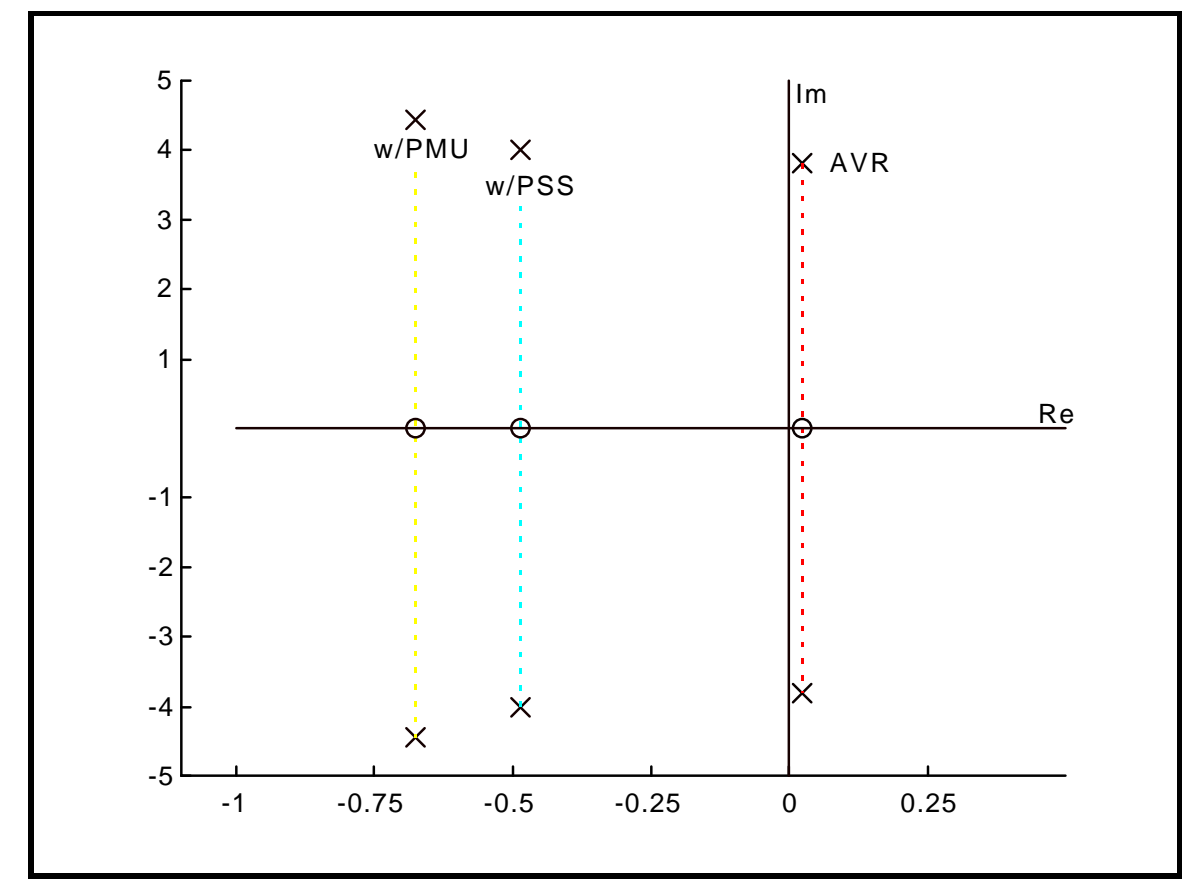

Figure 6.1: Mode Improvement Plot

The complete oscillation profile resulting from the application of the one PSS/two PMU control structure with $\mathrm{K}_{\mathrm{S}}=15.5$ for the base case system is shown in Table 6.7. The only difference between Table 6.7 and the others dealing with oscillation profiles is that the second column, which provides the PSS/PMU location, indicates the following: the first number is the generator with the PSS; the second is the location of the remote measurements. As with the others, the mode, frequency, damping, dominant states and mode shape are indicated. Finally, with the modification of the gain, an increase in the participation factors up to 0.866 for Area Two is realized. One possible conclusion is that the influence of the 1-PSS/2-PMU structure has been increased. Also, the influence of the remote feedback control structure remains above that of the PSS alone since the damping ratio improves from 0.131 to 0.1327 .

\begin{tabular}{|c|c|c|c|c|}
\hline $\begin{array}{c}\text { \# of } \\
\text { PMUs }\end{array}$ & $\begin{array}{c}\text { Location } \\
\text { GEN }\end{array}$ & Eigenvalue & $\begin{array}{c}\text { Frequency } \\
\text { Hz }\end{array}$ & $\begin{array}{c}\text { Damping } \\
\text { Ratio }\end{array}$ \\
\hline 2 & $\mathbf{1 1 - 1}$ & $-\mathbf{- 0 . 6 7 7 0} \pm \mathrm{j} 4.442$ & $\mathbf{0 . 7 0 7 0}$ & $\mathbf{0 . 1 5 0 7}$ \\
& $11-2$ & $-0.5946 \pm \mathrm{j} 4.248$ & 0.6761 & 0.1386 \\
& $12-1$ & $-0.4503 \pm \mathrm{j} 4.610$ & 0.7337 & 0.0972 \\
& $12-2$ & $-0.4655 \pm \mathrm{j} 4.429$ & 0.7049 & 0.1045 \\
& $1-11$ & $-0.6018 \pm \mathrm{j} 4.410$ & 0.7018 & 0.1352 \\
& $1-12$ & $-0.5241 \pm \mathrm{j} 4.389$ & 0.6986 & 0.1186 \\
& $2-11$ & $-0.5438 \pm \mathrm{j} 4.351$ & 0.6925 & 0.1240 \\
& $2-12$ & $-0.4794 \pm \mathrm{j} 4.310$ & 0.6859 & 0.1106 \\
\hline
\end{tabular}

Table 6.7: Oscillation Profile for Accelerating Power Input 


\subsubsection{Robustness Analysis and Secondary Tuning}

To determine if the tuning of the PSS with remote feedback was robust, the operating point of the system was varied over a wide range and the small signal stability investigated. The first test consisted in using a continuation power flow method [39]. This test involved increasing Ptie by increasing the active power of the load on Bus 13 until a conjugate pair of eigenvalues transversely crossed the imaginary axis, signifying an angular instability. This is called a Hopf Bifurcation Point [37]. However, this point could not be determined under the topology given, due to the limit of voltage collapse. The voltage collapse limit is attained when one of the eigenvalues becomes zero, indicating that the Jacobian matrix has become singular. To significantly increase the voltage collapse limit so the angular oscillations occur first, the impedances of the two lines between Buses 3 and 13 were decreased. The results of these tests are given in Table 6.8. The first column indicates the impedance ratio value, where Z-Line is equal to the impedance of one of the lines from Bus 3 to Bus 13 and Z/2 one-half, Z/4 is one quarter, etc. The next four columns give the load at Bus 13 needed to create the corresponding Ptie in the four following columns. The "Max" column indicates the maximum values for which the load flow converges, or where the Jacobian matrix is non-singular, at each impedance. The "AVR" column gives the values attainable with only AVRs on the generators (no PSSs or PMUs). The "w/PSS" column indicates the values for a PSS on GEN11, its previously determined optimal location. The "w/PMU" column gives the values for the same optimally located 1-PSS/2-PMU structure on GEN11 and GEN1 as previously discussed.

\begin{tabular}{|c|c|c|c|c|c|c|c|c|}
\hline Z-Line & Max & $\begin{array}{c}\text { Bus 13 } \\
\text { AVR }\end{array}$ & $\begin{array}{l}\text { Load } \\
\text { w/PSS }\end{array}$ & $\begin{array}{c}\text { (MW) } \\
\text { w/PMU }\end{array}$ & Max & $\begin{array}{l}\text { Ptie } \\
\text { AVR }\end{array}$ & $\begin{array}{c}\text { (MW) } \\
\text { w/PSS }\end{array}$ & w/PMU \\
\hline Z & 1937 & 1634 & 1937 & 1937 & 558 & 248 & 558 & 558 \\
\hline Z/2 & 2196 & 1841 & 2196 & 2196 & 820 & 456 & 820 & 820 \\
\hline$Z / 4$ & 2464 & 2307 & 2464 & 2464 & 1092 & 926 & 1092 & 1092 \\
\hline Z/8 & 2699 & 2380 & 2368 & 2393 & 1328 & 998 & 984 & 1010 \\
\hline$Z / 10$ & 2764 & 2358 & 2352 & 2368 & 1396 & 974 & 968 & 984 \\
\hline $\mathrm{Z} / 12$ & 2812 & 2346 & 2344 & 2356 & 1444 & 962 & 960 & 972 \\
\hline Z/14 & 2850 & 2339 & 2339 & 2348 & 1482 & 956 & 956 & 964 \\
\hline $\mathrm{Z} / 16$ & 2880 & 2334 & 2336 & 2342 & 1512 & 950 & 952 & 958 \\
\hline
\end{tabular}

Table 6.8: Line Impedance Variation Test Results

Until the lines are shortened beyond Z-Line $=\mathrm{Z} / 8$, the voltage collapse limit is exceeded before the angular stability limit is passed. Under these conditions, both the PSS and 1-PSS/2-PMU structures provide an increase in the Ptie critical value to the point where the angular instability is reached. Beginning with an impedance of $\mathrm{Z} / 8$, the differences between the different types of control become evident. These results are shown in Figures 6.2, 6.3 and 6.4.

In Figure 6.2, the Bus-13 Load stability limits for the AVR, w/PSS and w/PMU control structures are shown for impedance values of Z-Line/4 and smaller (Z/8, Z/10, Z/12, Z/14 and Z/16). It is clearly evident that the 1-PSS/2-PMU structure provides the best results. The Ptie-stability limit 
curve is depicted in Figure 6.3. Again, the improvement of the response of the remote feedback controller is evident. Finally, in Figure 6.4 the entire Ptie vs. Z-Line curve is shown, including the voltage collapse limit, to illustrate the response of the different controllers over the entire test range.

For these results, it should be noted that the remote feedback PSS was retuned at the Z/8 line impedance case. Frequency response analysis was used to estimate the new values for an additional lead-lag control block based on the similarity of its response with regard to the previous controller and the resulting stability of the inter-area mode. When added, this block provided the stable response over the entire operating point range where Z-Line was smaller than Z/8; however, to ensure that this controller was robust, the response of the system was investigated under the operating conditions involving impedance values of Z-Line $=\mathrm{Z} / 4$ and larger.

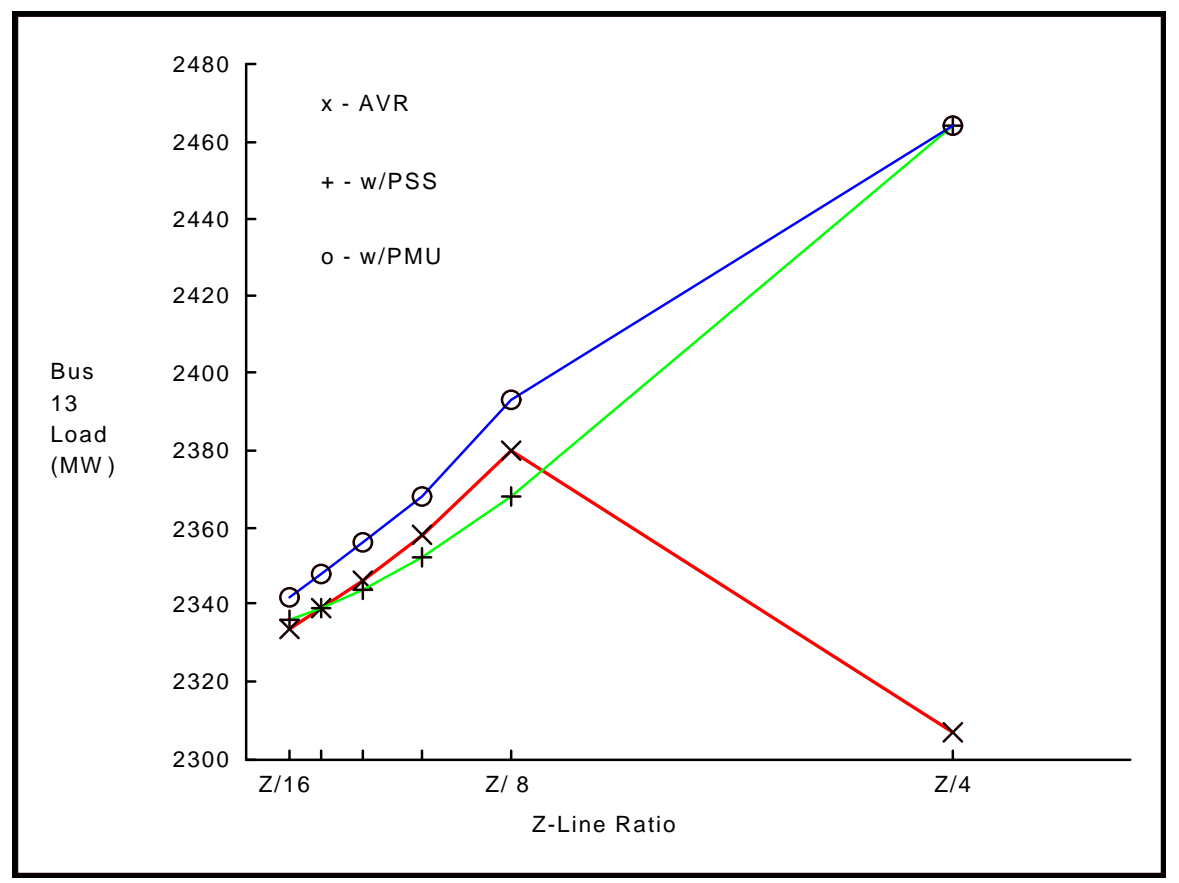

Figure 6.2: Bus 13 Load vs. Z-Line 


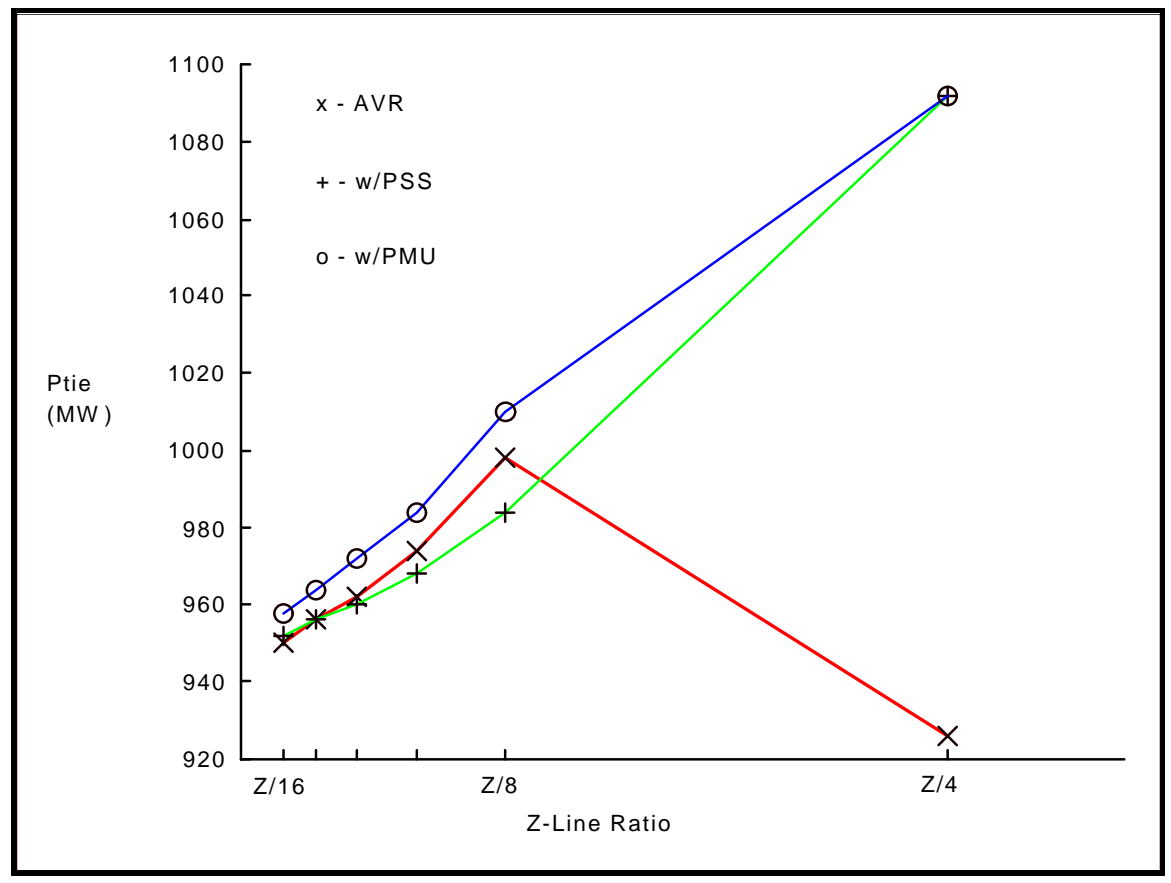

Figure 6.3: Ptie vs. Z-Line

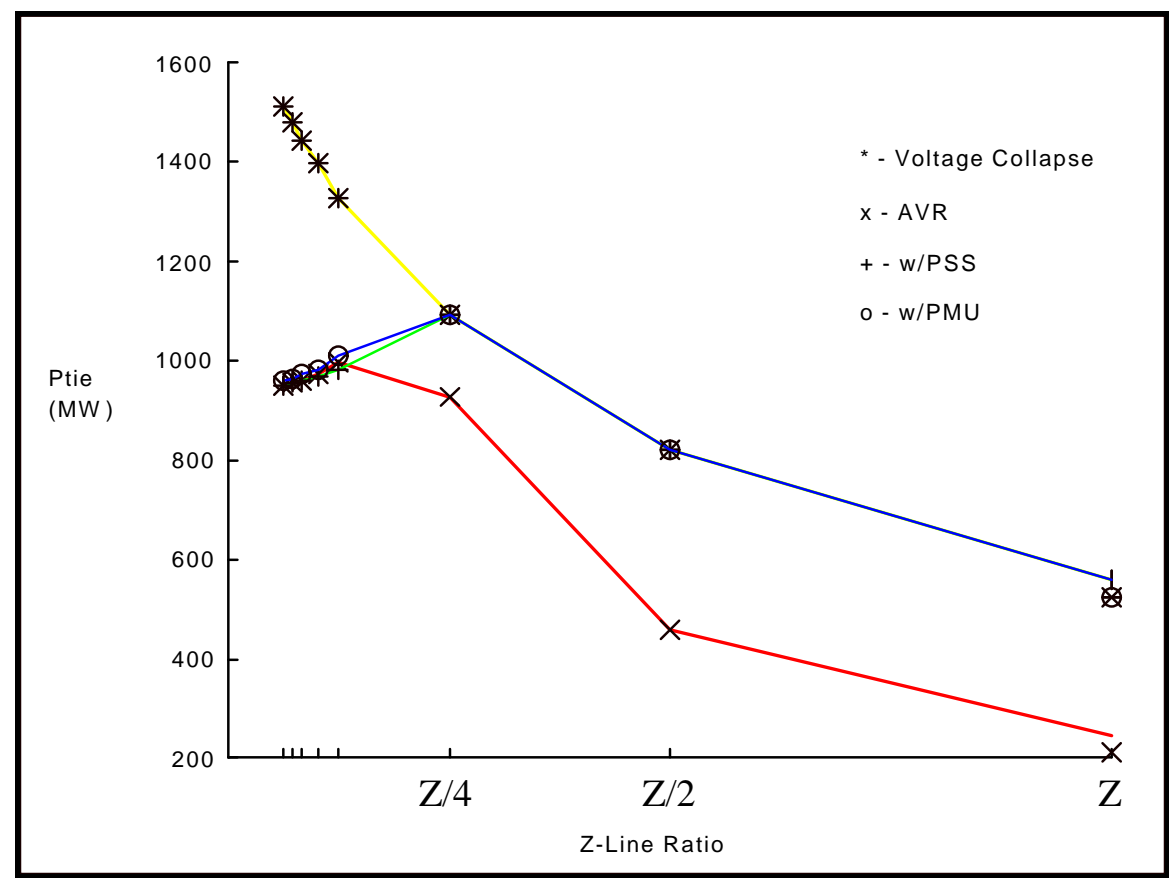

Figure 6.4: Complete Ptie vs. Z-Line 
After the retuning, the inter-area mode itself approaches the angular stability limit with respect to the original single lead-lag tuning, yet the eigenvalues remain in the left-hand side of the complex plane. Under these conditions, the plot would be identical to the one given in Figure 6.4 for line impedance values greater than Z/8. The PSS tuning values for both the local and remote feedback controllers in this thesis are given in Table 6.9. The "PSS" tuning is the values determined for the accelerating power PSS by Bourlès et al. [26]. The "1-PSS/2-PMU" tuning is the parameter set called the first approximation, showing the gain modification. The final set is the one with the additional lead-lag block, as discussed in this section.

\begin{tabular}{|c|c|c|c|}
\hline Parameter & PSS & 1-PSS/2-PMU & New PSS/PMU \\
\hline $\mathrm{K}_{\mathrm{S}}$ & 11.3 & 15.5 & 15.5 \\
$\mathrm{~T}_{1}$ & 0.1432 & 0.1432 & 0.1432 \\
$\mathrm{~T}_{2}$ & 2.1511 & 2.1511 & 2.1511 \\
$\mathrm{~T}_{3}$ & 10.0 & 10.0 & 0.625 \\
$\mathrm{~T}_{4}$ & 10.0 & 10.0 & 10.0 \\
$\mathrm{~T}_{5}$ & 30.0 & 30.0 & 30.0 \\
VPSS $_{\max }$ & 0.055 & 0.055 & 0.055 \\
VPSS $_{\min }$ & -0.055 & -0.055 & -0.055 \\
\hline
\end{tabular}

Table 6.9: PSS Tuning

\subsection{Time-Domain Response}

To evaluate the response of the remote feedback structure under severe operating point changes, such as a short-circuit or sudden load change, time-domain simulations were performed using EUROSTAG. The cases will be described, followed by a description of the plots of results from the cases, and finally the plots themselves.

Recalling the system topology from Figure 5.5, the first case is a short-circuit in the system on the line between Bus 3 and Bus 102. The duration of the fault is $100 \mathrm{~ms}$, with an additional 100ms delay before the line is placed back into service. This test provides a look at the oscillation damping provided by the PSS in the system under transient stability conditions. The second case is a the addition of $150 \mathrm{MW}$ of load at Bus 13. This test provides a look at the voltage and power response characteristics in the system under transient conditions. Both tests began under the base case loading condition; that is, a load of $1767 \mathrm{MW}$ at Bus 13 and Ptie = $382 \mathrm{MW}$.

Figure 6.5 shows the coherent response and damping of the oscillation between GEN1 and GEN11 under the short-circuit case. While at first this response seems more severe than that of the PSS alone of Figure 6.6, it should be noted that a coherent response indicates that the generator rotors are not hunting for synchronism. The rotor angle variation for GEN1 and GEN11 is displayed for both control structures in Figures 6.7 and 6.8. The improved response in terms of minimal rotor angle swing are quite noticeable with the 1-PSS/2-PMU structure. 
For the load addition case, the generator terminal voltage is shown for all of the control configurations in Figure 6.9, while Figure 6.10 shows the terminal voltage angle response. Again, the smaller variation of the generator terminal voltage affected with the 1-PSS/2-PMU structure is quite noticeable versus that of the PSS alone. In Figure 6.10, the relative lack of oscillation in the response of the 1-PSS/2-PMU structure is evident versus that of the PSS alone. Figure 6.11 shows the voltage angle difference between Bus 3 and Bus 13 under the load addition case for the 1-PSS/2-PMU and single PSS structures. The advantage of the PMUs, seen as the lack of oscillation in the voltage angle, is quite remarkable. Finally, the active power swing by GEN11 is shown in Figure 6.12. The well damped power oscillation is clearly shown for the 1-PSS/2-PMU structure versus the single PSS alone.

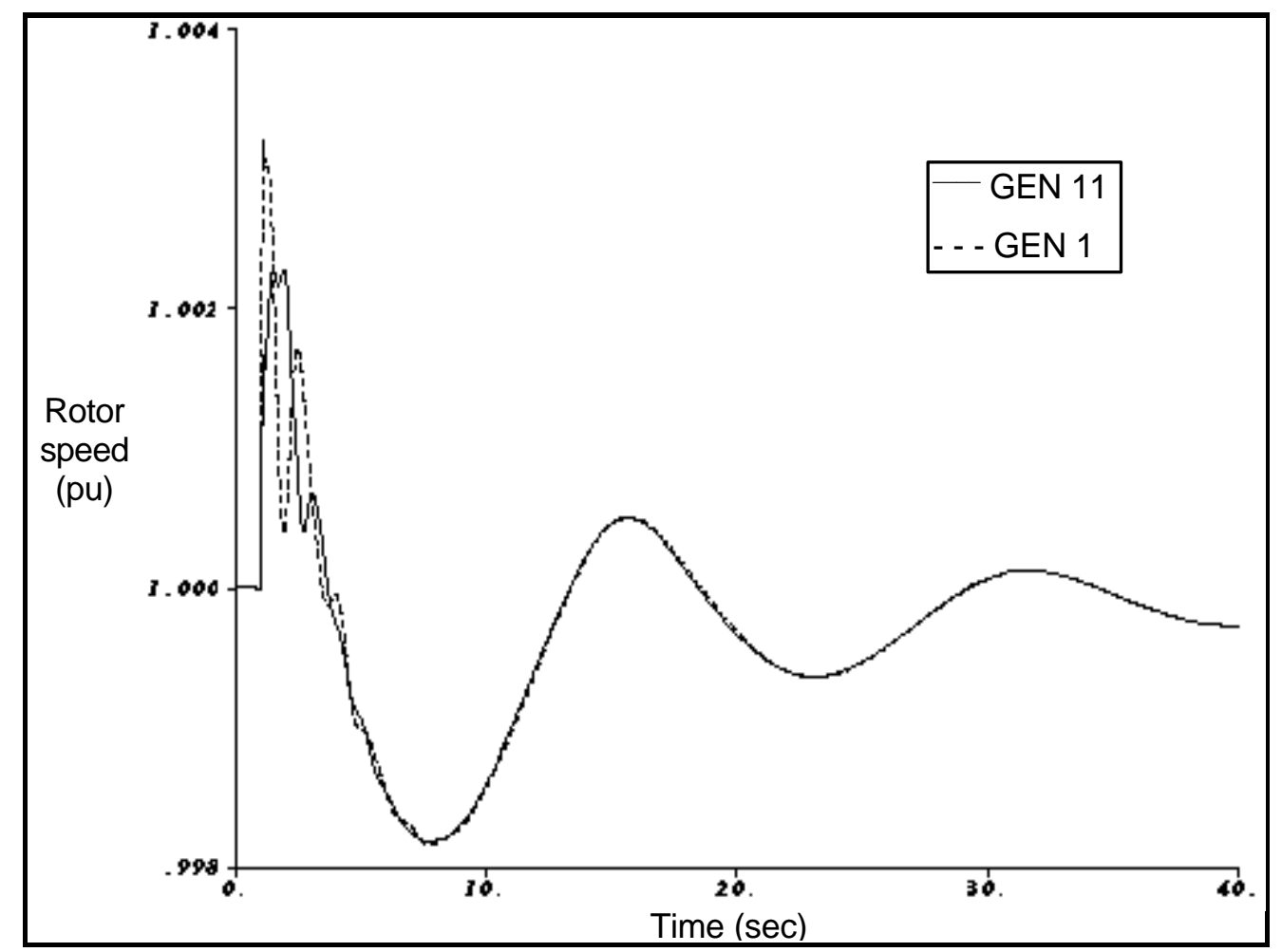

Figure 6.5: Rotor Speed Oscillations for the 1-PSS/2-PMU Structure 


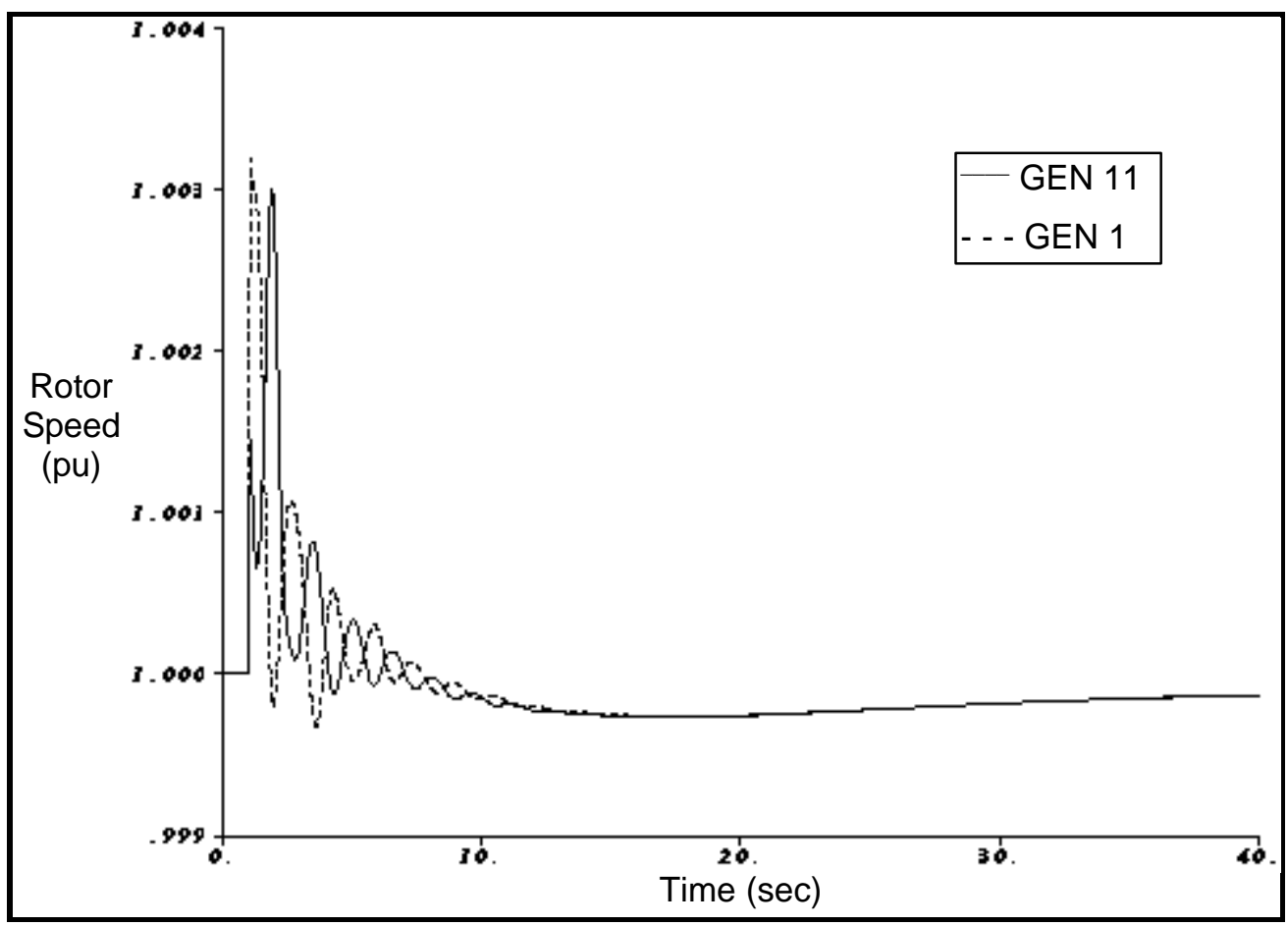

Figure 6.6: Rotor Speed Oscillations for the Local Feedback PSS

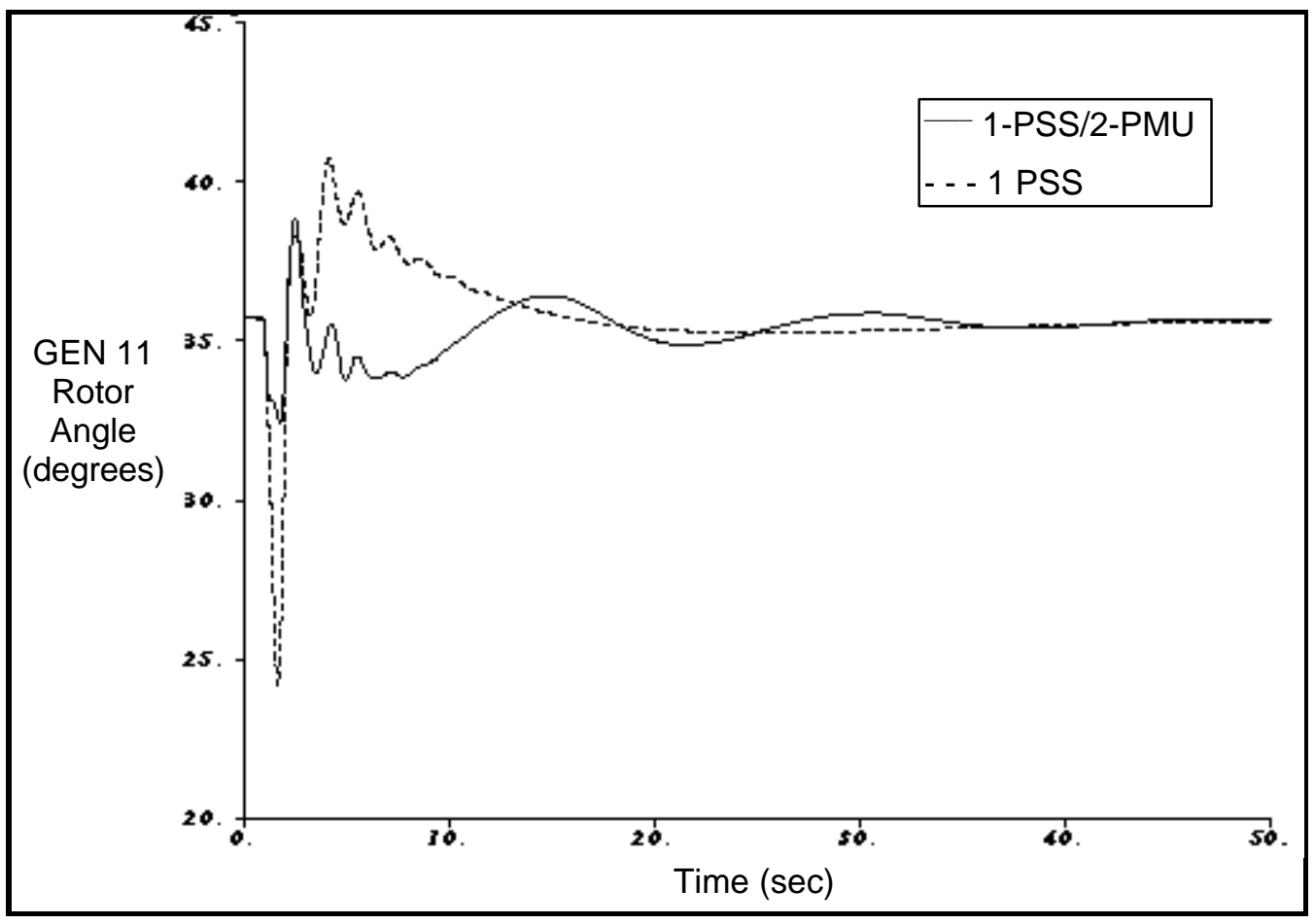

Figure 6.7: Rotor Angle Variation for GEN11 


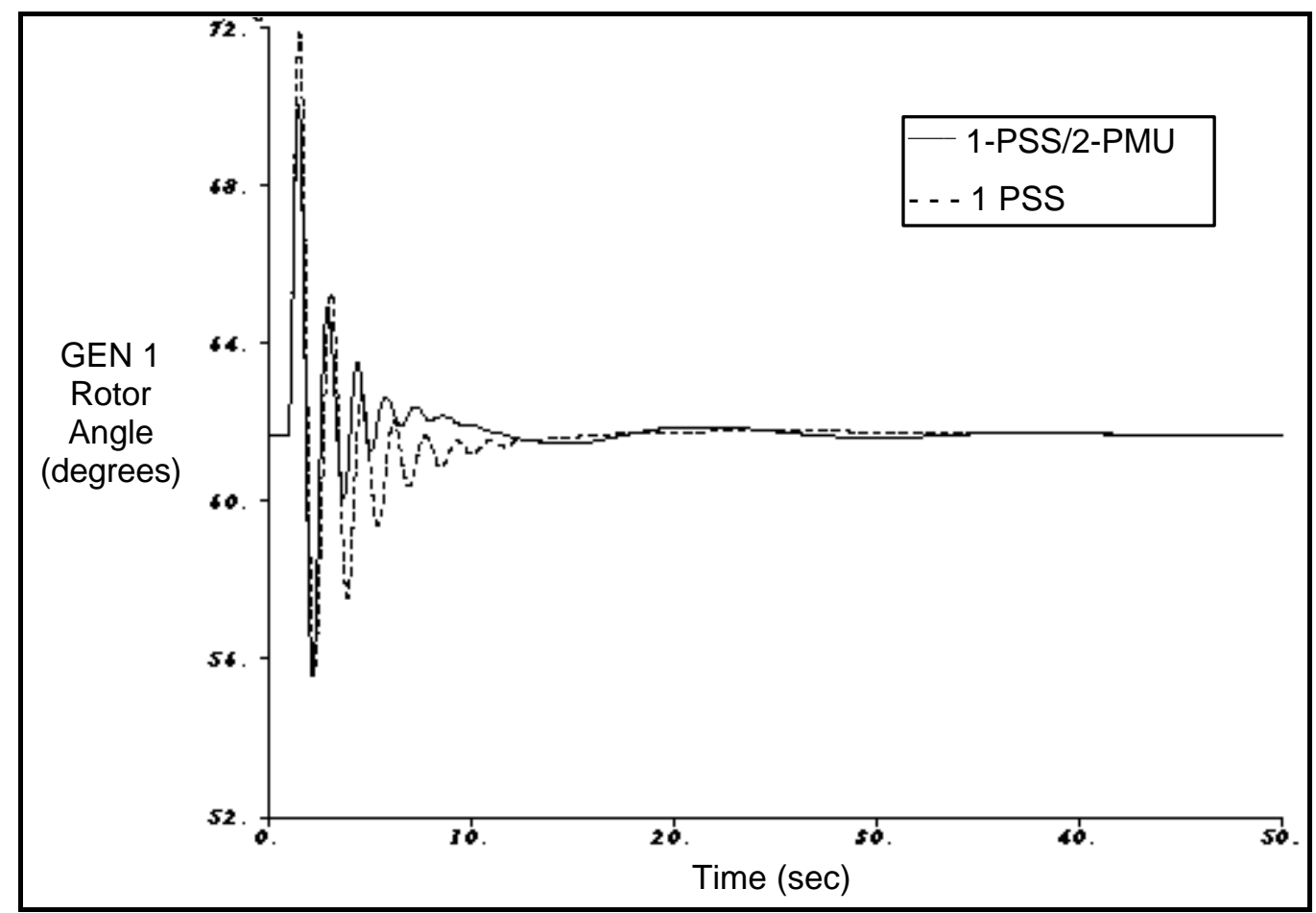

Figure 6.8: Rotor Angle Variation for GEN1

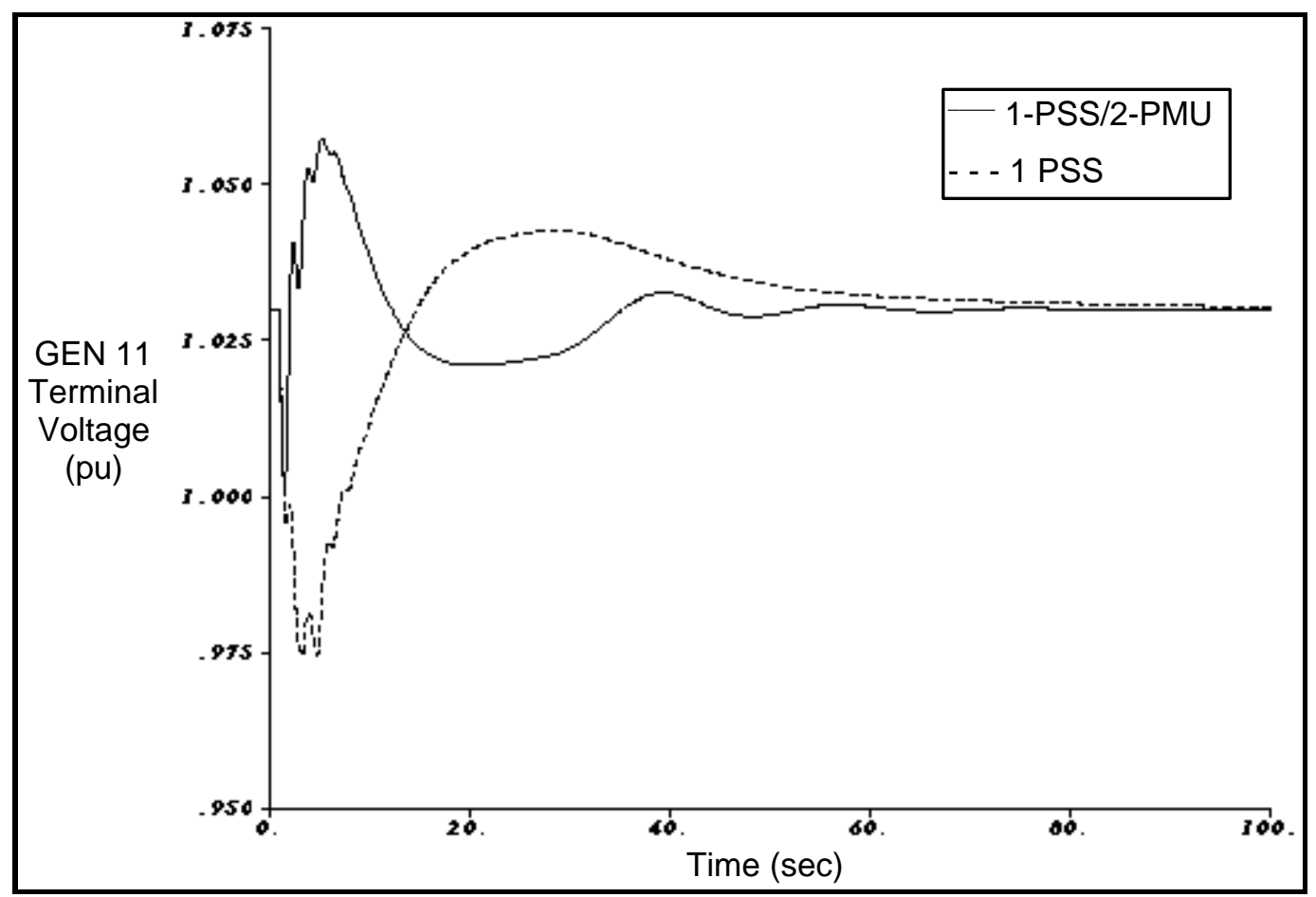

Figure 6.9: Comparative Terminal Voltage for GEN11 


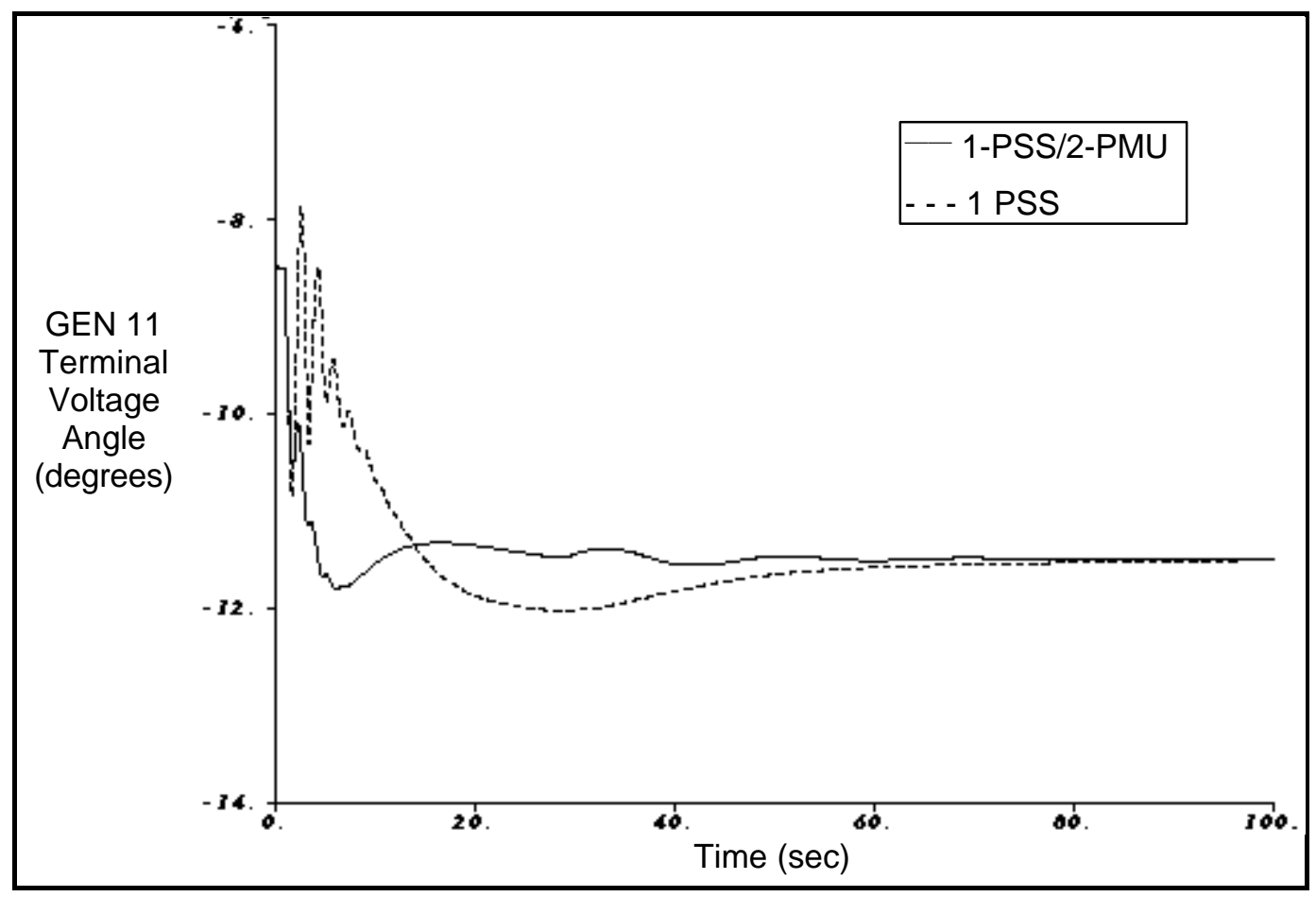

Figure 6.10: Comparative Terminal Voltage Angles for GEN11

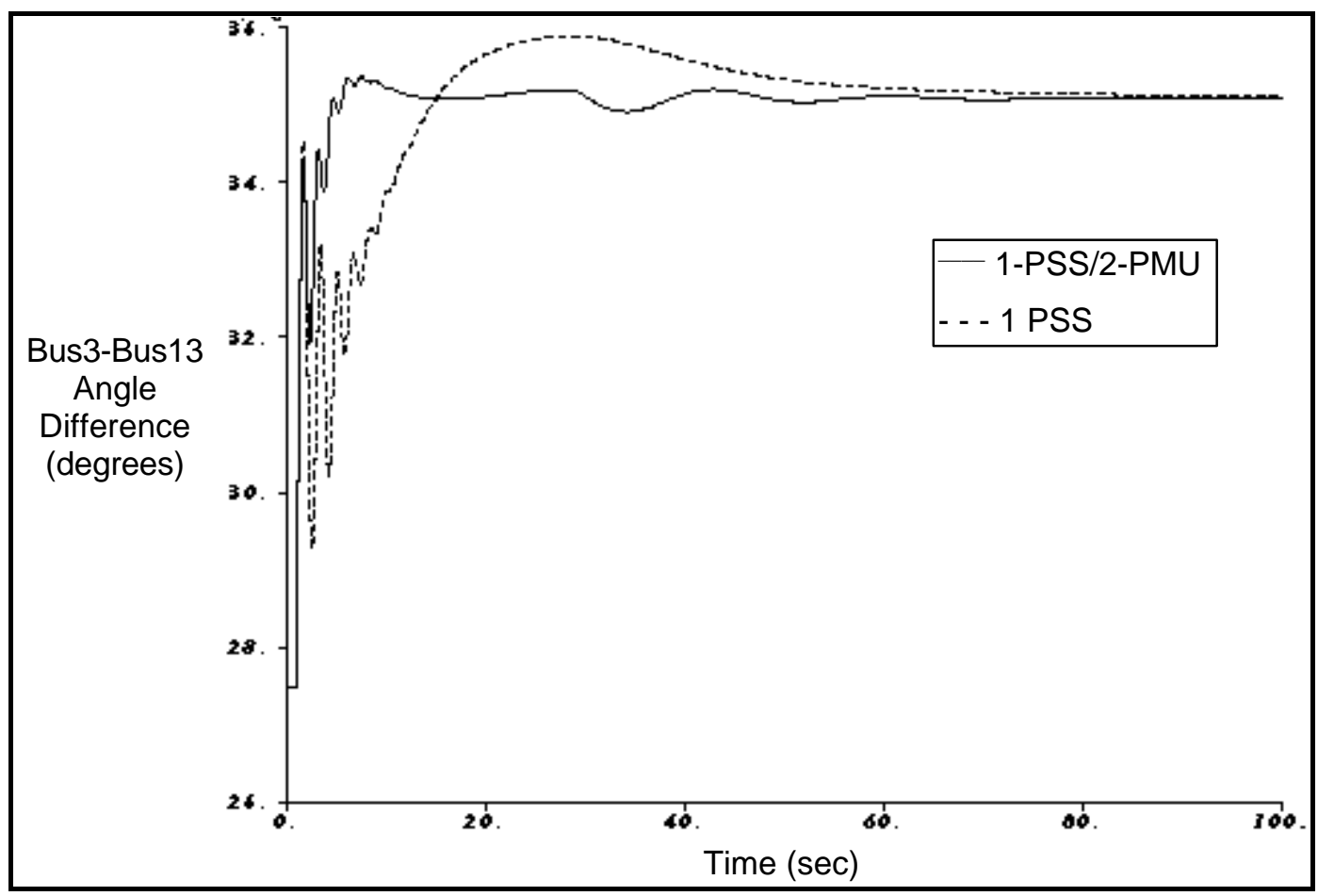

Figure 6.11: Angle Response Across Tie-Line 3-13 


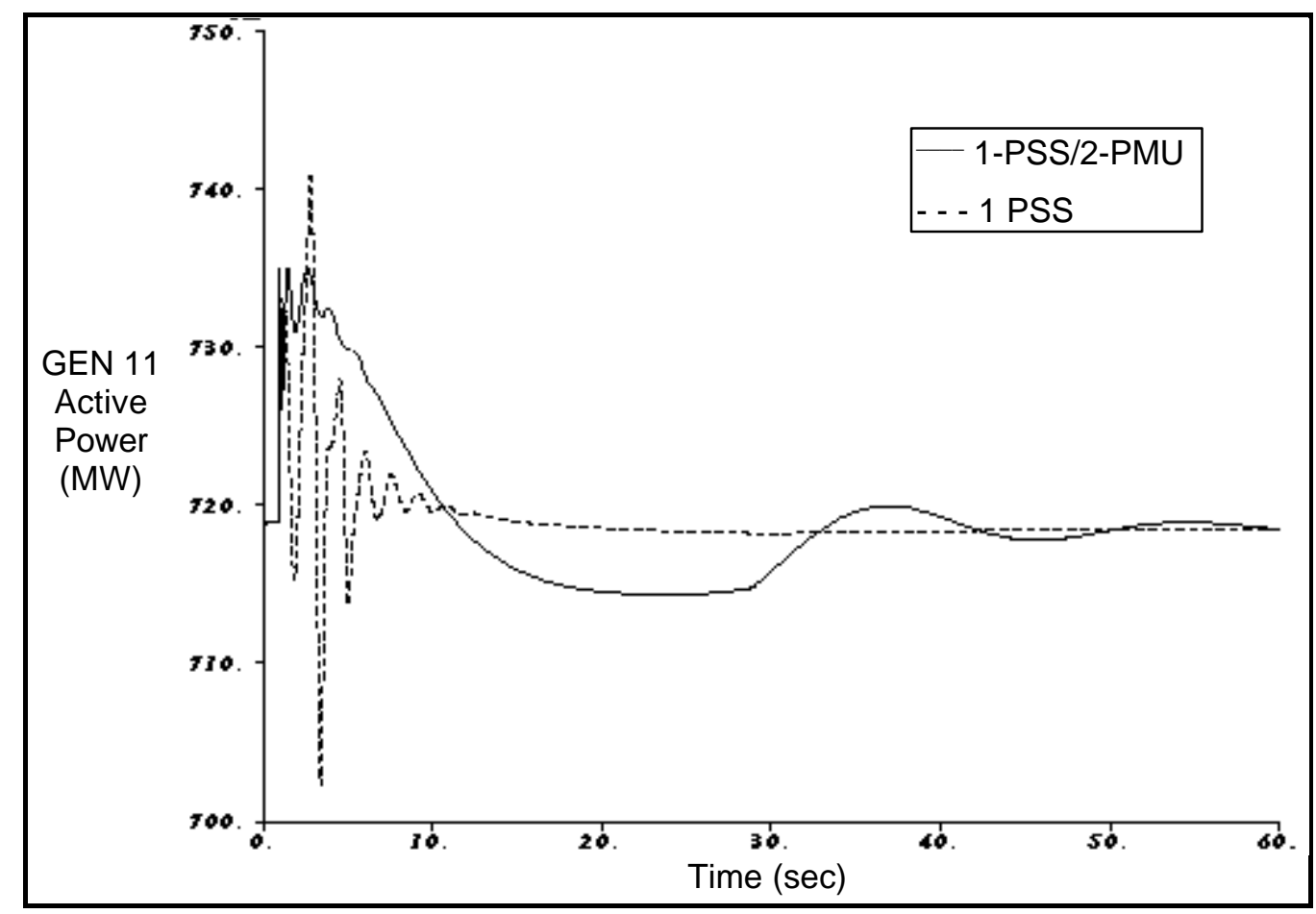

Figure 6.12: GEN 11 Active Power 


\section{7}

\section{CONCLUSIONS}

\subsection{Summary of Results}

As stated in Section 1.2, the focus of this thesis was the damping of inter-area oscillations using remote synchronized phasor measurement feedback control of a power system stabilizer on a twoarea, 4-machine test system. The purpose was to provide a more robust control over a large excursion of the operating point versus an optimal local controller. A methodology to site the PMUs in the power system based on eigenanalysis was proposed and tested. The resulting placement and tuning of the remote feedback controller and PMUs resulted in the increased damping and stability, and improved controllability/observability of the inter-area mode of interest. The modes in the system were also found to be decoupled; that is, the tuning proposed for one type of mode did not destabilized the other modes in the system. The proposed controller was shown to be robust throughout a broad range of the test system operating conditions. It was also shown that the tie-line power in the test system could be increased without loss of stability. Finally, time-domain simulations involving a short-circuit and load addition in the test system confirmed that the proposed 1-PSS/2-PMU control structure provides an improvement over the conventional control structure.

\subsection{Future Work}

The future of this particular research topic has many different facets. These include the study of different power system stabilizer designs, different input signals, communications network design and construction and coordinated power system device control. Each of these is dealt with separately in the following sections.

\subsubsection{Other PSS Controller Designs}

Besides the "lead-lag" structure investigated in this thesis, there are several existing and proposed controller designs. The "4-loop" combined AVR/PSS controller used by Electricité de France [26] and a recently investigated proportional-integral-derivative (PID) PSS design [27] have both shown promising results. Different in their feedback loops, transfer functions or tuning methods, 
both designs have been examined on a one-machine, infinite bus or a small test system. Their application on larger systems has not yet been reported.

\subsubsection{Other PSS Input Signals}

Current research has focused on rotor speed as a control variable [1,21,22 23-25,27]. A frequency-input controller $(\Delta \mathrm{f})$ has been investigated as well. However, it has been found that frequency is highly sensitive to the strength of the transmission system, that is, more sensitive when the system is weaker, which may offset the controller action on the electrical torque of the machine [23]. Other limitations include the presence of sudden phase shifts following rapid transients and large noise induced by industrial loads [11]. On the other hand, the frequency signal is more sensitive to inter-area oscillations than the speed signal, and may contribute to better oscillation attenuation [23-25]. Besides the PSS controller, a frequency-based FACTS device has been advocated for power oscillation damping; however, the frequency metered values are provided by measurements other than SPMs [14].

\subsubsection{Time Delay}

It is well-documented that delay compensation is an important consideration for feedback systems, especially those with remote measurements involved $[1,4,9,10,13]$. The delay time is a direct function of the communications network for the remote signal. Microwave (Japan), hybrid fiber-optic/satellite (France) and traditional phone network (U.S.A.) systems are in existence at the present time. The remote feedback applications involved have either an optimized and dedicated transmission system (Japan), adaptive delay-sensitive programming (France) or are research applications (U.S.A.). As such, the use of this type of real-time control system proposed in this thesis has yet to be installed in a power system. For the moment, the cost of a dedicated high-speed fiber-optic communications system to diminish signal transmission delay time is not a feasible control option for most power system utilities $[9,10]$. However, preliminary investigation of the problem of inter-area oscillation damping using remote measurements revealed that a small amount of delay (up to 200ms) did not significantly reduce the performance of the response of the system under small-signal or transient conditions during time-domain simulations [38].

\subsubsection{FACTS Devices and Coordinated Systems}

As mentioned in Section 7.2.2, FACTS devices can be employed to provide power oscillation damping [14]. In particular, the thyristor-controlled series compensator (TCSC) provides two levels of power system modification to allow the operator to maximize the stability of the system. Used to add reactance in series, this device provides the means to lower line impedance, yielding greater power transits when necessary. A second capability is the ability to quickly damp power flow oscillations through the modulation of the control of the compensators.

One possible control structure could involve PSSs placed to damp local modes of oscillation present in the system and a TCSC with synchronized frequency measurements to provide the damping for any inter-area oscillations in the system. The presence of the PMU-type 
synchronized measuring devices for this application would also allow their use for other applications such as out-of-step relaying and fault detection and elimination, as proposed in $[2,5,8,14]$. 